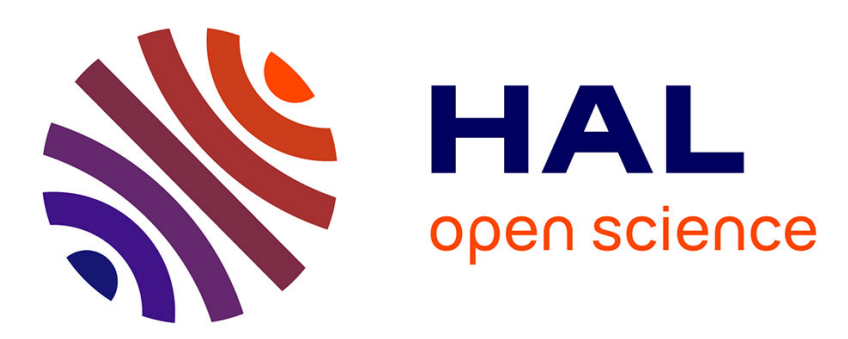

\title{
Criteria and Convergence Rates in Noisy Optimization
} Sandra Astete-Morales, Marie-Liesse Cauwet, Olivier Teytaud

\section{To cite this version:}

Sandra Astete-Morales, Marie-Liesse Cauwet, Olivier Teytaud. Criteria and Convergence Rates in Noisy Optimization. Genetic and Evolutionary Computation Conference (GECCO 2015), Jul 2015, Madrid, Spain. 2015, 10.1145/2739482.2764722 . hal-01217128v2

\section{HAL Id: hal-01217128 https://hal.inria.fr/hal-01217128v2}

Submitted on 19 Jan 2016

HAL is a multi-disciplinary open access archive for the deposit and dissemination of scientific research documents, whether they are published or not. The documents may come from teaching and research institutions in France or abroad, or from public or private research centers.
L'archive ouverte pluridisciplinaire HAL, est destinée au dépôt et à la diffusion de documents scientifiques de niveau recherche, publiés ou non, émanant des établissements d'enseignement et de recherche français ou étrangers, des laboratoires publics ou privés. 


\section{Criteria and Convergence Rates in Noisy Optimization}

\author{
Sandra Astete-Morales \\ TAO, Inria, Lri, Umr Cnrs 8623 \\ Bat. 650, Univ. Paris-Sud \\ 91405 Orsay Cedex, France \\ sandra-cecilia.astete- \\ morales@inria.fr
}

\author{
Marie-Liesse Cauwet \\ TAO, Inria, Lri, Umr Cnrs 8623 \\ Bat. 650, Univ. Paris-Sud \\ 91405 Orsay Cedex, France \\ marie- \\ liesse.cauwet@inria.fr
}

\author{
Olivier Teytaud \\ TAO, Inria, Lri, Umr Cnrs 8623 \\ Bat. 650, Univ. Paris-Sud \\ 91405 Orsay Cedex, France \\ olivier.teytaud@inria.fr
}

\begin{abstract}
In an optimization framework, some criteria might be more relevant than others; the internal computational cost of the optimization algorithm might be negligible or not; the quality of intermediate search points might be important or not. For this reason measuring the performance of an algorithm is a delicate task. In addition, the usual criteria are often approximated for the sake of simplicity of the analysis, or for simplifying the design of test beds. This situation makes sense both in noise-free and noisy settings; however it is more often crucial in the latter case. We here discuss and compare several performance criteria published in the literature in the case of noisy optimization. We review existing rates, for various existing criteria, propose new rates, and check if some classically observed criteria are good approximations of sound criteria.
\end{abstract}

\section{Categories and Subject Descriptors}

G.1.6 [Optimization]: Unconstrained optimization

\section{Keywords}

Noisy optimization

\section{FRAMEWORK AND CRITERIA}

Given a fitness function $F: D \in \mathbb{R}^{d} \rightarrow \mathbb{R}$, also known as objective function, optimization (minimization) is the search for the optimum point $x^{*}$ such that $\forall x \in D, F\left(x^{*}\right) \leq F(x)$. The fitness function may be corrupted by noise. A common case of noise is the additive noise case. Given a search point $x \in D$, evaluating $F$ in $x$ results in an altered fitness value $f(x, w)$ as follows:

$$
f(x, w)=F(x)+w,
$$

where $w$ is an independent random variable of mean zero and variance $\sigma$. An optimization algorithm generates $\left(x_{n}\right)_{n \geq 1}$, successive search points at which the objective function is evaluated - in a noisy manner. It can also generate $\left(\tilde{x}_{n}\right)_{n \geq 1}$

Permission to make digital or hard copies of part or all of this work for personal or classroom use is granted without fee provided that copies are not made or distributed for profit or commercial advantage and that copies bear this notice and the full citation on the first page. Copyrights for third-party components of this work must be honored. For all other uses, contact the owner/author(s).

GECCO '15 July 11-15, 2015, Madrid, Spain

(C) 2016 Copyright held by the owner/author(s).

ACM ISBN 978-1-4503-3488-4/15/07.

DOI: http://dx.doi.org/10.1145/2739482.2764722 which are recommendations or approximations of the optimum. $\tilde{x}_{n}$ is provided after $n$ fitness evaluations are performed.

[Simple Regret] $(S R)$ :

$$
S R_{n}=F\left(\tilde{x}_{n}\right)-F\left(x^{*}\right) .
$$

[Approximate Simple Regret] $(A S R)$ :

$$
A S R_{n}=\min _{m \leq n} F\left(x_{m}\right)-F\left(x^{*}\right) .
$$

[Robust Simple Regret] $(R S R)$ :

$$
R S R_{n}=\min _{k \leq n} \max _{k-g(k)<m \leq k}\left(F\left(\tilde{x}_{m}\right)-F\left(x^{*}\right)\right),
$$

where $g(n)$ is a polylogarithmic function of $n$.

[Cumulative Regret] $(C R)$ :

$$
C R_{n}=\sum_{i=1}^{n}\left(F\left(x_{i}\right)-F\left(x^{*}\right)\right) .
$$

[Average Regret] $(A R)$ :

$$
A R_{n}=\frac{1}{n} C R_{n}
$$

We define the corresponding "slope" of the various regrets introduced previously by:

$$
s(* R)=\limsup _{n \rightarrow \infty} \log \left(* R_{n}\right) / \log (n),
$$

where $* R$ stands for $S R, A S R, R S R, C R$ or $A R$ and $\log$ is the natural logarithm. From now on, $s($.$) stands for "slope$ of". The slope is a random variable; however in many cases it is almost surely equal to some constant. We also defined the slope in expectation, as follows:

$$
s^{\mathbb{E}}(* R)=\limsup _{n \rightarrow \infty} \log \left(\mathbb{E}\left(* R_{n}\right)\right) / \log (n) .
$$

\section{CONCLUSION}

In this paper we analyse several criteria for the performance of algorithms in noisy optimization problems. We provide several rates for each of the criteria and compare them. Some are rigorously proved, others are conjectured. Table 1 summarizes our results.

Simple regret. From theory, it appears that Evolution strategies, when correctly tuned in terms of resamplings, essentially reach half the speed of classical noisy optimization 


\begin{tabular}{|c|c|c|c|c|}
\hline Algorithm & $\begin{array}{c}\text { Slope of the } \\
\text { simple regret } \\
s(S R)\end{array}$ & $\begin{array}{c}\text { Slope of the } \\
\text { approximate } \\
\text { simple regret } \\
s(A S R)\end{array}$ & $\begin{array}{c}\text { Slope of the } \\
\text { robust } \\
\text { simple regret } \\
s(R S R)\end{array}$ & $\begin{array}{c}\text { Slope of the } \\
\text { average } \\
\text { regret } \\
s(A R) \\
\end{array}$ \\
\hline Random search & 0 & $-\frac{2}{d}$ & $-\frac{2}{d}$ & 0 \\
\hline $\mathrm{ES}$ & 0 & $-\frac{2}{d}$ & $-\frac{2}{d}$ & 0 \\
\hline$E S+$ resampling & $-\frac{1}{2} \sharp$ & $-\frac{1}{2}$ & $-\frac{1}{2} \sharp$ & $-\frac{1}{2}^{\sharp}$ \\
\hline Modified $E S+$ resampling & $-\frac{1}{2}^{\sharp}$ & $-\frac{1}{2}-\frac{2}{d}^{\sharp}$ & $-\frac{1}{2}-\frac{2}{d} \sharp$ & $-\frac{1}{2}^{\sharp}$ \\
\hline $\begin{array}{l}\text { Shamir (original) } \\
\text { Shamir (adapted for } A S R \text { ) }\end{array}$ & \begin{tabular}{|l|}
$-1^{\dagger}$ \\
$-1^{\dagger}$ \\
\end{tabular} & \begin{tabular}{|l|l|}
$0^{\dagger}$ & and $0^{*}$ \\
& $-1^{\dagger}$ \\
\end{tabular} & \begin{tabular}{|l|}
$-1^{\dagger}$ \\
$-1^{\dagger}$ \\
\end{tabular} & \begin{tabular}{|l|}
0 \\
0 \\
\end{tabular} \\
\hline $\begin{array}{l}\text { Fabian (original) } \\
\text { Fabian (adapted for } A S R \text { ) }\end{array}$ & \begin{tabular}{|c|}
$-(1-e)^{*}$ \\
$-(1-e)^{*}$
\end{tabular} & $\frac{-e^{\prime *}}{-(1-e)^{*}}$ & $\frac{-(1-e)^{*}}{-(1-e)^{*}}$ & $-e^{\prime *}$ \\
\hline Fabian (adapted for $A R$ ) & & & & $-\frac{1}{2}+e^{*}$ \\
\hline
\end{tabular}

Table 1: Table of regrets for various algorithms. Rates with $\sharp$ means that the convergence is with high probability; for numbers with $\dagger$, the convergence is in expectation and the convergence is a.s ones with $*$. When nothing is specified, the convergence holds for the 3 different types. Boxed results are proved and others are conjectured.

algorithms in terms of simple regret, on the log-log scale. This corresponds to a squared computation time.

Cumulative regret. In terms of $C R$, however, they reach the same rate as noisy optimization algorithms, e.g. as Fabian's algorithm with a proper choice of constants ([1] considers mainly simple regret, but their result implies optimal rates for $C R$ as well, for a proper choice of parameters), or the best rate in [2].

Approximations. The $A S R$ is a poor approximation of simple regret since: (i) A fast algorithm for $S R$ can have a bad rate for $A S R$ (see e.g. Fabian and Shamir algorithm). However, this can be solved by modifying the algorithm in order to sample, sometimes, a recommended point. (ii) A slow algorithm for $S R$ can have a fast rate for $A S R$, and this is a more serious problem. There is no simple patch for that. Test beds using $A S R$ will overestimate algorithms which include random exploration. This is partially, but not totally, solved by $R S R$. However, the difference decreases with the dimension for most algorithms. Indeed, $A S R$ is a better estimate of $A R$ than an estimate of $S R$, as in many cases $s(A S R)=s(A R)$, except for algorithms which are specifically adapted for $A S R$. Incidentally, $A S R$ was proposed in the evolutionary computation community, and $A R$ (or, equivalently, $C R$ ) is the criterion for which evolutionary computation performs best in noisy optimization.
We did not come up with a satisfactory criterion, which would be consistent with $S R$ (at least, same slope) and without drawbacks as those presented above, except the simple regret itself. The drawback of $S R$ is that it is not necessarily non-increasing, which is an issue for the concept of "first hitting time". Another further work is the refinement of the theoretical analysis. We have compared slopes, but in some cases we have slopes for almost sure convergence, in other cases slope with high probability, and in others slope in expectation.

\section{REFERENCES}

[1] V. Fabian. Stochastic Approximation of Minima with Improved Asymptotic Speed. Annals of Mathematical statistics, 38:191-200, 1967.

[2] O. Shamir. On the complexity of bandit and derivative-free stochastic convex optimization. In COLT 2013 - The 26th Annual Conference on Learning Theory, June 12-14, 2013, Princeton University, NJ, $U S A$, pages 3-24, 2013. 